\title{
Signal-processing software for teaching and research in psychoacoustics under UNIX and X-Windows
}

\author{
JOHN F. CULLING \\ MRC Institute of Hearing Research, University Park, Nottingham, England
}

\begin{abstract}
A package of software is described that generates, analyzes, stores, and displays sampled waveforms. The package is designed for use under UNIX and includes $\mathrm{C}$ source code, UNIX manual pages, and tutorial documents. The programs interact via UNIX pipes using an ASCII-text data format, which enables the user to view the data in numerical form as well as through the use of plotting programs. Among many other functions, the programs can do the following: efficiently generate linearphase FIR filters with arbitrary transfer functions; generate impulse responses for rectangular rooms of specified dimensions; convolve waveforms with each other; perform Fourier transformation and inverse Fourier transformation; filter waveforms in the Fourier domain; filter waveforms according to the peripheral frequency selectivity of the human auditory system; cross-correlate waveforms; autocorrelate waveforms; synthesize complex waveforms, including vowel sounds and white noise. The software can read and write a variety of commonly used waveform file formats. The data can be plotted on an X-Window display using the gnuplot software, which has been included in the package. The complete software package is available via anonymous ftp from ftp.ihr.mrc.ac.uk in $\sim \mathrm{ftp} / \mathrm{pub} / \mathrm{johncu} /$ wave.tar.Z
\end{abstract}

Psychoacoustic experimentation has traditionally been conducted using large collections of complex hardware. The advent of inexpensive computers and accurate digital-to-analog converters has involved the computer in the stimulus-generation process, and increases in computer power now allow complex auditory modeling to be performed. Such modeling requires that the stimuli to be modeled be available to the computer in digital form, and a fair comparison between human and model requires that identical stimuli be used in both experiment and model. The simplest way of achieving this goal is to complete the entire stimulus-generation process within the computer. In the near future, we may expect the only necessary external hardware to be an amplifier and a pair of headphones. This paper describes a coherent suite of digital signal-processing (DSP) software for use in the psychoacoustic laboratory. The software includes tools for stimulus generation and for psychoacoustic modeling.

This package would have been impossible to assemble without the technical advice and support of Andrew Sidwell, David Marshall, and Tim Folkard. Without Thomas Williams' and Colin Kelley's free and portable plotting program, "gnuplot," a publicly distributed version of the package would have been of limited use. The software for reading and writing Sun/NeXT audio-files was written by Peter Kabal of McGill University. The author would like to thank Quentin Summerfield, Peter Assmann, John Foster, Greg Sandell, and Alain de Cheveigné for their comments on the software and/or this manuscript. Correspondence should be addressed to J. F. Culling, University Laboratory of Physiology, Parks Rd., Oxford OX1 3PT, England (e-mail: jfc@physiol.ox.ac.uk).

\section{Design Features}

The inspiration for this signal-processing package came mainly from the data analysis software developed by Perlman (1980). Hence, where Perlman called his software package "|STAT" (pronounced "unix-stat" or "pipestat"), the present package is named "WWVE." Perlman wrote programs in C (Kernighan \& Ritchie, 1978), which could be used in exactly the same way as UNIX's own utilities; the programs behave as though they are part of the UNIX operating system. Readers who are unfamiliar with UNIX and the use of "pipes" to communicate information between its utilities are referred to Perlman's article for a brief introduction. Information passes from the "standard output" of one program into the "standard input" of another, via a pipe (written "|"). In this way, successive programs can be chained together in order to apply a sequence of transformations before sending the data to a disk file or to a plotting program. For instance, using |WAVE, "gaussian 1024 | fft | plot" generates 1,024 points of Gaussian noise, performs a fast Fourier transform, and then plots the resulting frequency spectrum on the screen.

A crucial feature of $\mid$ WAVE is that the data pass from one modular program to another through a UNIX pipe in a simple ASCII-text format, preceded by some ASCII header data. This scheme has four advantages. (1) The use of UNIX pipes enables any user with UNIX experience to quickly master the use of the programs and to combine them in novel ways without programming and without learning a new user interface. (2) The user can then immediately write UNIX scripts to generate stimuli for whole experiments to program a sequence of model- 
ing processes or to run a model on a whole set of stimuli. (3) The use of ASCII-text makes the data humanreadable so that the user has direct access to both the header information and the numerical data simply by piping the output into the standard UNIX utility more, which displays ASCII text a page at a time. This feature is vital for teaching purposes, and it prevents the unwelcome occurrence of binary data being accidentally piped to screen. (4) The simple, transparent data format maximizes the ease with which a $C$ programmer can, if necessary, write and debug an additional program that will complement those already included in the package.

Although the ASCII-text format used here is an inefficient way for the programs to communicate (compared with a binary data format), the processing times are generally determined by the signal processing within the programs.

\section{Comparison With Commercial Products}

Various commercial packages offer extensive DSP functions. These include ILS, MatLab, and ESPS. The principle differences between these products and IWAVE are that JWAVE is available free of charge and that it is directly oriented toward psychoacoustics. Consequently, |WAVE does not include many of the functions that engineers would expect from a more general DSP package, rather it contains those that are most useful for generating and analyzing psychoacoustic stimuli. Many of the programs and their command line options have been chosen to facilitate the particular requirements of psychoacousticians. The fbank program, for instance, is not a general filterbank program but specifically offers a simulation of the human peripheral auditory system, and $f f t$ similarly contains an option (-e) for producing cochlear excitation patterns.

WWAVE is also distinctive in its conformity to the UNIX principle of modular programs that communicate via pipes. It is very much a native UNIX package and will consequently appeal mainly to those who are already experienced UNIX users and who will appreciate the fact that they are not required to learn a new user interface or meta-language (however user-friendly it may be) in order to start using the system.

\section{Compatibility and Compilation}

|WAVE should compile on any UNIX system; the programs have been compiled and tested on a SUN SPARCstation, a NeXT, and a DEC System 5400. The package includes an appropriate UNIX "makefile" for convenient compilation.

The use of UNIX pipes is essential. Although it is possible to use pipes under DOS, many of the programs have large memory requirements and would be impossible to use without large areas of directly accessible memory, which DOS does not permit. A few of the programs (e.g., fir, room, and convolve) can be used without pipes and have small memory requirements. These programs are useful in their own right and should compile on almost any system with a C-compiler, but most of the software would require major modifications in order to port successfully to a DOS environment. |WAVE can be used on a non-UNIX machine by using an X-terminal emulation program and an EtherNet connection to a UNIX server; WAVE has been tested in this way using eXceed under Windows. X-Windows is used to provide high-quality graphics using gnuplot. However, gnuplot can be used with a wide variety of other graphics devices; only the PostScript laserprinter options have so far been incorporated into the software.

Unfortunately, there is no widely used standard for sampled-data file formats. The software therefore provides various binary audio-file formats that may be selected at compilation time. In this way, the compiled software adopts the local standard as its default file format. In addition, the $f d$ program can read data from disk in a number of unheadered formats. The file-format options that currently are fully supported include WFF, ILS, and Sun/NeXT. This list is likely to be extended in the future.

\section{Stimulus Generation}

The primary function of the programs is the generation of complex auditory stimuli. The system is sufficiently flexible that most stimuli can be generated in several different ways. Aside from the ability to use the programs in different combinations, several of the programs have been provided with extensive command-line options that can be used in complex combinations. In particular, the klatt, fir, ffilt, room, and $w d m^{1}$ programs offer many options. In these cases, facilities have been included that enable complex control parameters or long lists of command-line arguments to be read from an ASCII file or from a pipe.

It is advisable to create stimuli using UNIX scripts. In this way, a small number of nested scripts and parameter files form a "recipe" that can generate (or regenerate) the stimuli for a whole experiment.

\section{Modeling}

Modern computer models of auditory processes invariably begin with a filtering process that simulates the frequency selectivity of the peripheral auditory system. The package provides this facility through $f$ bank, which uses the gamma-tone filter-bank software developed by Patterson, Nimmo-Smith, Holdsworth, and Rice (1987, 1988). Further processes include the Meddis (1986, 1988) haircell model (an option in fbank), which provides an estimate of the probability of a neural discharge on an auditory nerve fiber, cross-correlation (using the jeffress program), which is frequently used in models of binaural interaction (Colburn, 1973, 1977; Jeffress, 1972), and autocorrelation (using autocorr), which has been used in models of pitch perception (Licklider, 1951; Meddis \& Hewitt, 1991a, 1991b). In addition, the $w d m$ program provides a more open-ended opportunity to manipulate the data through algebraic expressions. 


\section{Teaching}

The use of modular programs and the human-readability of data have particular advantages for teaching. This is best illustrated by an example. Many people have difficulty with the concept of Fourier transformation. The programs could be used to help a student form an understanding and familiarity with this essential technique. For the purpose of psychoacoustics, a detailed understanding of the underlying mathematics of Fourier transformation is unnecessary, but a thorough understanding of the general concept (and its applications) is important. Using /WAVE, it is possible to demonstrate how sounds can be transformed into the frequency domain without any loss of information, modified if desired, and then returned to the time domain. The student can explore the transformations achieved by the programs, viewing the output produced at various stages either in numerical form or using the plotting programs. For instance, a $1000-\mathrm{Hz}$ sine wave can be generated using the program klatt, and the student can inspect the wave with the command "klatt -hf 1000 | plot." The Fourier spectrum can be viewed with "klatt -hf $1000 \mid \mathrm{fft}$-p | plot." The "-p" tells $f f t$ to produce the phase spectrum as well as the power spectrum. The inverse transform can then be demonstrated with "klatt -hf 1000 | fft -p | ift | plot," to reproduce the original waveform. The student can view the information that is used by each program along the way.

The concept of Fourier transformation for sine waves of different frequencies is quite straightforward, but what about complex waveforms? The same process can be applied to an amplitude-modulated wave, illustrating how the frequency domain encodes the temporal changes (as side components) and showing that the frequency spectra form a completely reciprocal representation. Because all the information communicated by the programs is visible, the student can see that the spectra (power and phase) contain all that is needed by the inverse transform to recreate the original waveform.

\section{The Programs}

The following is a brief description of the 30 most important programs in the package. Space precludes these descriptions from being exhaustive either in terms of describing all the programs (there are currently 41 ) or in reporting all but the most useful of the features that they offer. The programs are listed alphabetically.

\begin{tabular}{|c|c|}
\hline add & $\begin{array}{l}\text { Reads in a list of waveform files supplied on } \\
\text { the command line, sums the waveforms that } \\
\text { they contain, and writes a new waveform file. } \\
\text { The relative levels of the signals in the new } \\
\text { file can be controlled. }\end{array}$ \\
\hline pend & $\begin{array}{l}\text { Concatenates a list of waveform files sup- } \\
\text { plied on the command line and writes them to } \\
\text { a new file. }\end{array}$ \\
\hline corr & $\begin{array}{l}\text { Performs running autocorrelation on the } \\
\text { waveform in each channel (Licklider, 1951). }\end{array}$ \\
\hline$d$ & $\begin{array}{l}\text { Removes the header from the incoming wave- } \\
\text { form and outputs the unheadered ASCII data. }\end{array}$ \\
\hline
\end{tabular}

This program is useful for putting data into statistical packages such as "ISTAT" (Perlman, 1980). For instance, the Pearson's $r$ correlation between the two channels of a stereo file can be found using Perlman's pair program by the following command: " $\mathrm{fd}$ stereofile | behead | pair."

cascade Accepts multiple waveforms from a pipe and orders them into a "waterfall" for plotting. The spacing, overlap, and scaling of the individual plots can be controlled. Hidden lines can be removed for a 3-D landscape effect. The different channels can also be pooled to form a summary plot, which can be used in conjunction with the fbank, autocorr, and jeffress programs to model pitch perception (Meddis \& Hewitt, 1991a, 1991b) and the auditory lateralization of sounds (Shackleton, Meddis, \& Hewitt, 1992). See Figures 3 and 4.

convert Converts files in a range of commonly used waveform file formats (ILS, WFF, NIST SPHERE, Sun/NeXT, DEC, IRCAM, INRS, and ESPS) into the default format (WFF, ILS, or Sun/NeXT).

convolve Convolves a waveform (from file or pipe) with an FIR filter that is stored in a file.

erbrate Converts between frequency values in hertz and frequencies in the psychophysical frequency scale, "equivalent rectangular bandwidths" (or ERB; see Moore \& Glasberg, 1983).

extract Accepts waveforms from the standard input and extracts specified portions of those waveforms for output.

fbank Filters waveforms on the standard input by a bank of "auditory" filters (Patterson \& Moore, 1986; Patterson et al., 1987), which have passbands and shapes that match the frequency selectivity of the peripheral auditory system (Moore \& Glasberg, 1983; Patterson \& Moore, 1986). Each output waveform can also by processed by the Meddis $(1986,1988)$ model of peripheral neural transduction. See Figures 3 and 4.

fd "Float-dumps" a waveform from a disk file onto the standard output, so that the data may be piped into other programs. By default, the waveform should be in the local site's standard file format (as specified at compile time), but a variety of headerless formats $(1-, 2-$, and 4-byte integer, 4- and 8-byte floating point, and ASCII) can be specified using commandline arguments. A header may be prefixed to the output in order to pipe the data into other |WAVE programs.

ffilt Filters the power and phase spectrum of a waveform in the Fourier domain. The program accepts Fourier data from a pipe (either complex spectra or power and phase spectra) and sends manipulated power and phase spectra to the standard output. ffilt can make manipulations that are specified as level changes, 
phase-shifts, or delays. These changes can be uniform across frequency or can have any specified frequency dependency. See Figure 2.

Performs Fourier transformation on waveforms on the standard input. The resulting transforms can be output as power spectra only, with the phase spectra as well, or in complex form. Hanning windowing can precede the analysis. The power spectrum can also be convolved by the frequency response of the peripheral auditory system to give a "cochlear excitation pattern" (Moore \& Glasberg, 1983, 1987). See Figures 1 and 2.

Generates a linear FIR filter using the hostwindowing technique (Abed \& Cain, 1978, 1984; Trinder, 1982). This method produces a mildly suboptimal linear FIR filter of any specification (within a dynamic range limitation of about $200 \mathrm{~dB}$ for a 512-point filter). The host-windowing technique is very fast compared with optimal methods such as the Remez exchange algorithm (Rabiner \& Gold, 1975). Filter cutoff slopes can be of the order of $1000 \mathrm{~dB} /$ oct.

gaussian Generates white noise using the method described by Klatt (1980) in which 16 successive random numbers, generated by the UNIX random number utility, are summed to form each output sample. The mean, skewness, and kurtosis are readily checked using the desc program from |STAT (e.g., "gaussian 10000 | behead | desc").

gnuformat Stores the waveform data and gnuplot commands in temporary files that can be read by gnuplot, which will use them to (1) write a PostScript file containing a plot of the data, (2) write a Hewlett-Packard graphics-language file containing a plot of the data, and (3) display a plot of the data on an X-Window (see Examples section below). The PostScript file is produced for the purpose of printing the plot. The Hewlett-Packard graphics-language file is produced, so that the graph may be loaded by a suitable graphics editor (see Examples section below).

hanning Applies a Hanning window or a series of overlapping Hanning windows to waveforms from a pipe. The latter function can be used to generate spectrograms in combination with fft (e.g., "klatt -k | hanning 10090 | fft | cascade -o 20 -h $\mid$ plot -b") and temporal envelopes using rms (e.g., "gaussian $1000 \mid$ hanning $5080 \mid$ rms -e (plot").

ift Performs an inverse Fourier transformation on complex or power plus phase spectra from a pipe.

jeffress Performs cross-correlation on stereo pairs of waveforms from a pipe, in order to model binaural interaction (Jeffress, 1972). See Figure 4. It can also perform equalization-cancellation, such as that proposed by Durlach's (1960) model of binaural masking release (see also Culling \& Summerfield, 1995; Durlach, 1972). klatt Synthesizes complex sounds, including vowels (Klatt, 1980), by sine-wave summation. A large number of options can control the frequencies, phases, and modulations of the fundamental, of individual harmonics, and of vowel formants. See Figure 1.

matplot Runs MatLab as a graphics and/or computational engine. Waveforms from a pipe are placed in a MatLab matrix called "W," and the user is given a MatLab environment in which to manipulate and plot the data. This program can only be used if MatLab is installed.

$p d p \quad$ Learns to recognize spectra (or other waveforms) presented to it on the standard input, using 2- or 3-layer PDP networks (Bechtel \& Abrahamson, 1991; Culling \& Darwin, 1994).

$r m s \quad$ Reports statistics of the waveform(s) from a pipe. Also produces spectrograms from an array of frequency channels, produced by fbank and temporal envelopes from time series that have been windowed using hanning.

roex Generates window shapes that are used to model the peripheral frequency selectivity (Patterson \& Moore, 1986) and temporal resolution (Plack \& Moore, 1990) of the human auditory system.

room Generates an impulse response between two points in a reverberant room with specified source and receiver locations, room dimensions, and wall absorbencies (Peterson, 1986). Head-shadow can also be simulated (Culling, Summerfield, \& Marshall, 1994).

store Saves waveforms from a pipe in a file with the local site's standard waveform file format.

unwrap Unwraps any phase spectra that arrive from a pipe.

$w d m \quad$ Performs arithmetic operations on waveform data from a pipe. The operations are specified by logical and algebraic expressions on the command line. In this way, waveforms may be added together, multiplied, log-compressed, and so on. For instance, gaussian $100 /$ fbank $-f 1000 / w d m$ "if $(x l>1)$ then $x 1$ else $l " /$ $w d m$ " $\log (x l) " /$ plot will plot the half-wave rectified and log compressed output of an auditory filter, tuned to $1 \mathrm{kHz}$, in response to a Gaussian noise.

$w f c \quad$ Combines a set of waveform files specified on the command line into a single multichannel file.

$w f s \quad$ Splits a specified waveform file into its component channels and writes those channels to a set of files with related names.

\section{Examples}

Figures $1-4$ show typical plots that can be produced using the programs. Each figure is titled with the command string that produced it. It is assumed in these example command strings (and strongly recommended to the user) that plot is defined using a UNIX alias as "gnuformat $\backslash !^{*}$; gnuplot .gnu_cmds." The figure captions give the total execution times for these commands on a SPARCstation 10/30. 


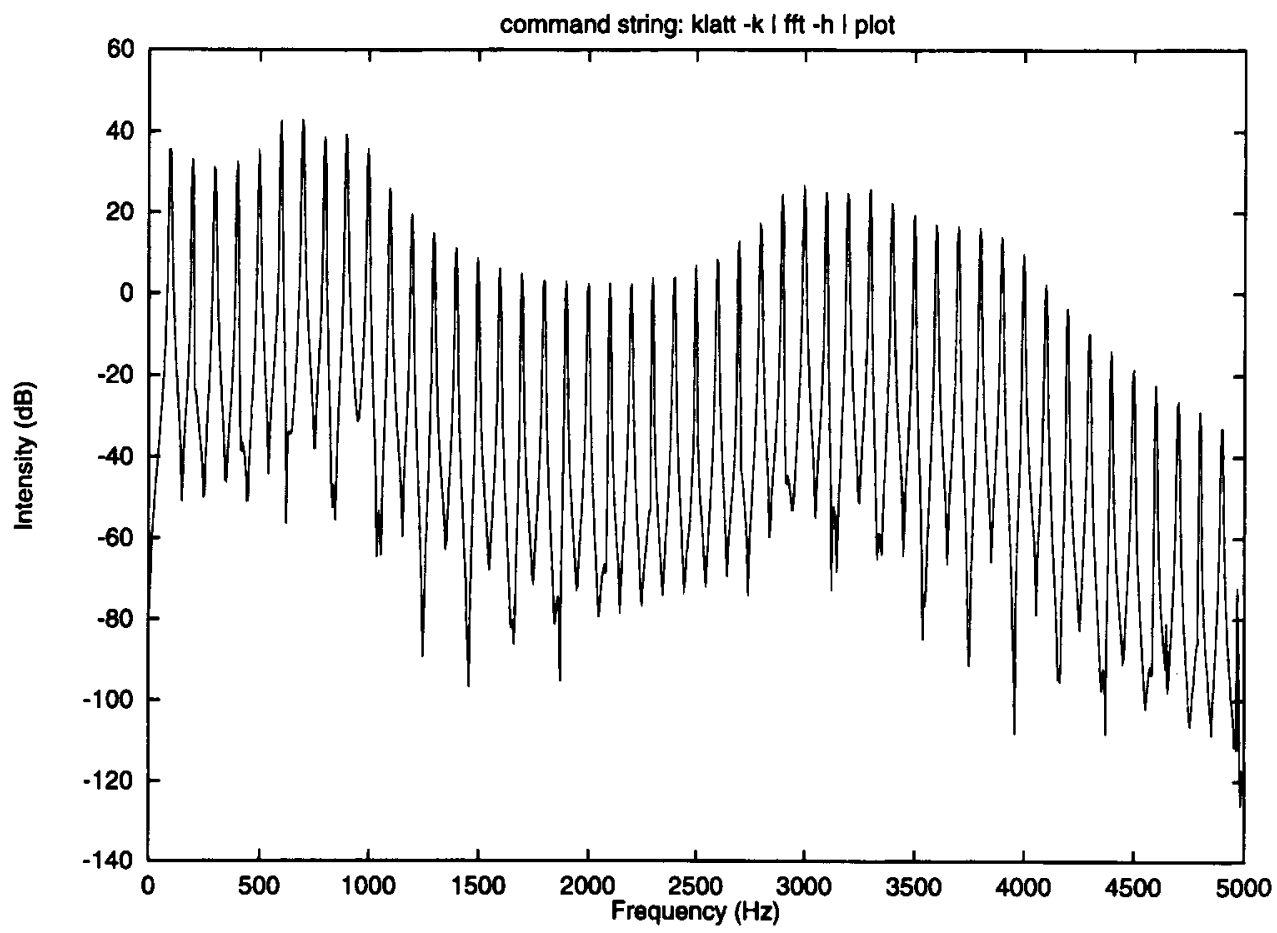

Figure 1. Fourier transform (power spectrum) of the default vowel sound (200 msec at a 10-kHz sampling rate of $/ \mathrm{a} /$ at $100 \mathrm{~Hz} F_{0}$ ) produced by klatt using a Hanning window. Execution time, 5 sec.

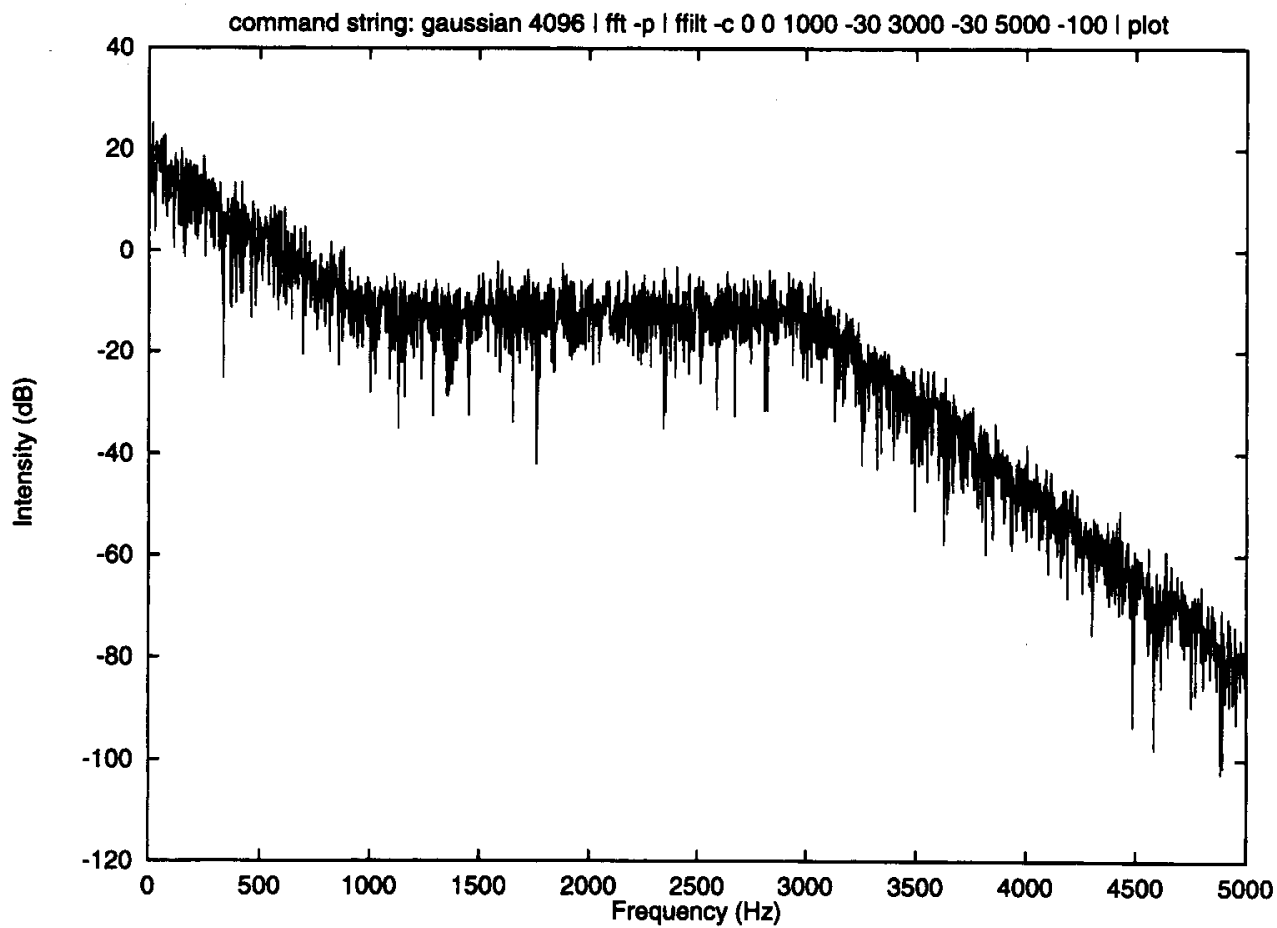

Figure 2. Fourier spectra (power only) of 4,096 samples of Gaussian noise, filtered in the Fourier domain to produce a ramped cutoff from 0 to $1 \mathrm{kHz}, 30-\mathrm{dB}$ attenuation between $1 \mathrm{kHz}$ and $3 \mathrm{kHz}$, and a ramped cutoff thereafter, which reaches $-100 \mathrm{~dB}$ at $5 \mathrm{kHz}$. Execution time, $8 \mathrm{sec}$. 


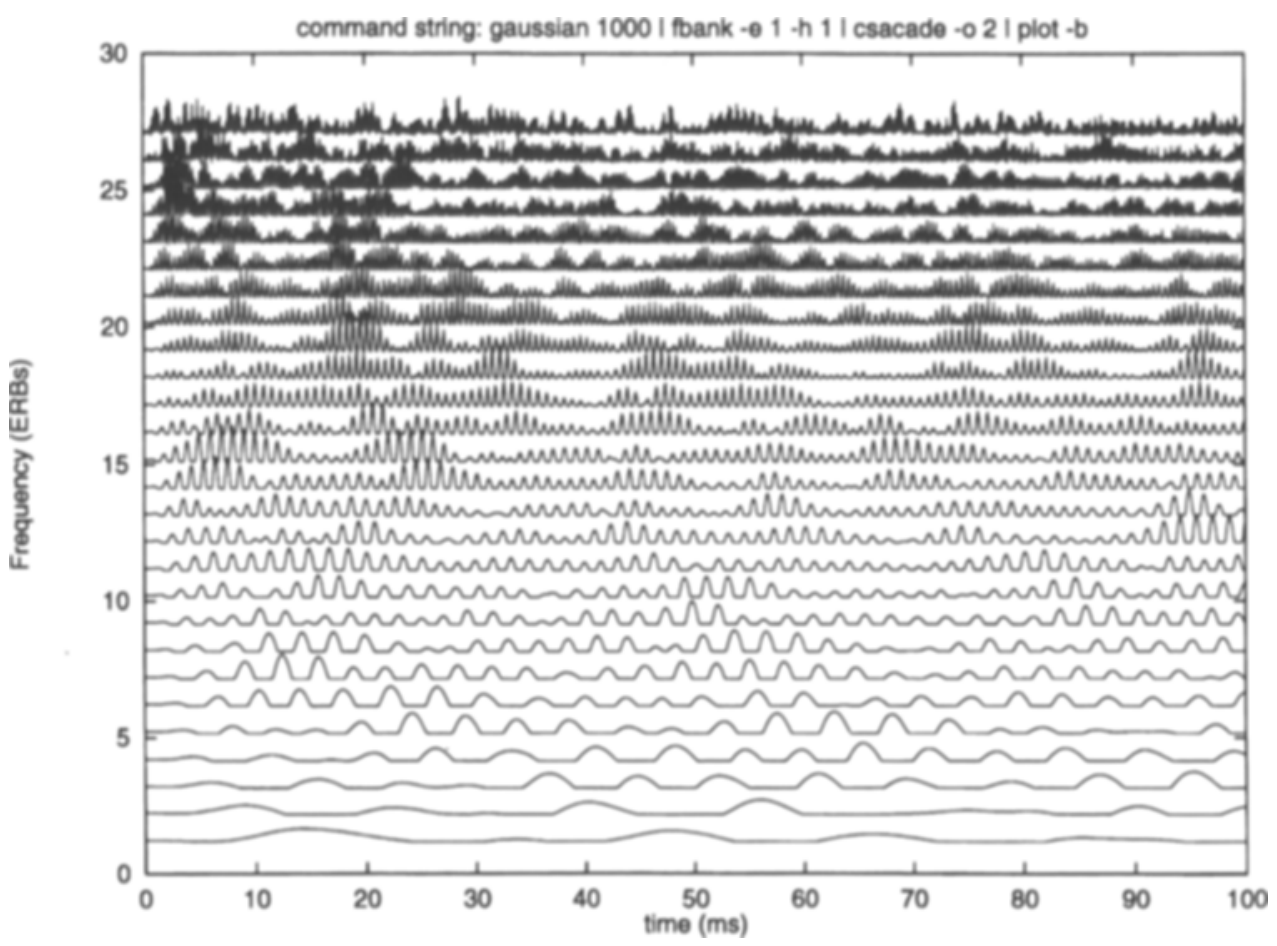

Figure 3. One thousand samples of Gaussian noise passed through a gamma-tone filterbank (Patterson et al., 1987, 1988) incorporating the Meddis $(1986,1988)$ haircell model with one frequency channel per ERB (Moore \& Glasberg, 1983). Each curve represents the predicted population response of (or probability of firing on) auditory nerve fibers tuned to the frequency (in ERBs) on the ordinate. Execution time, 25 sec.

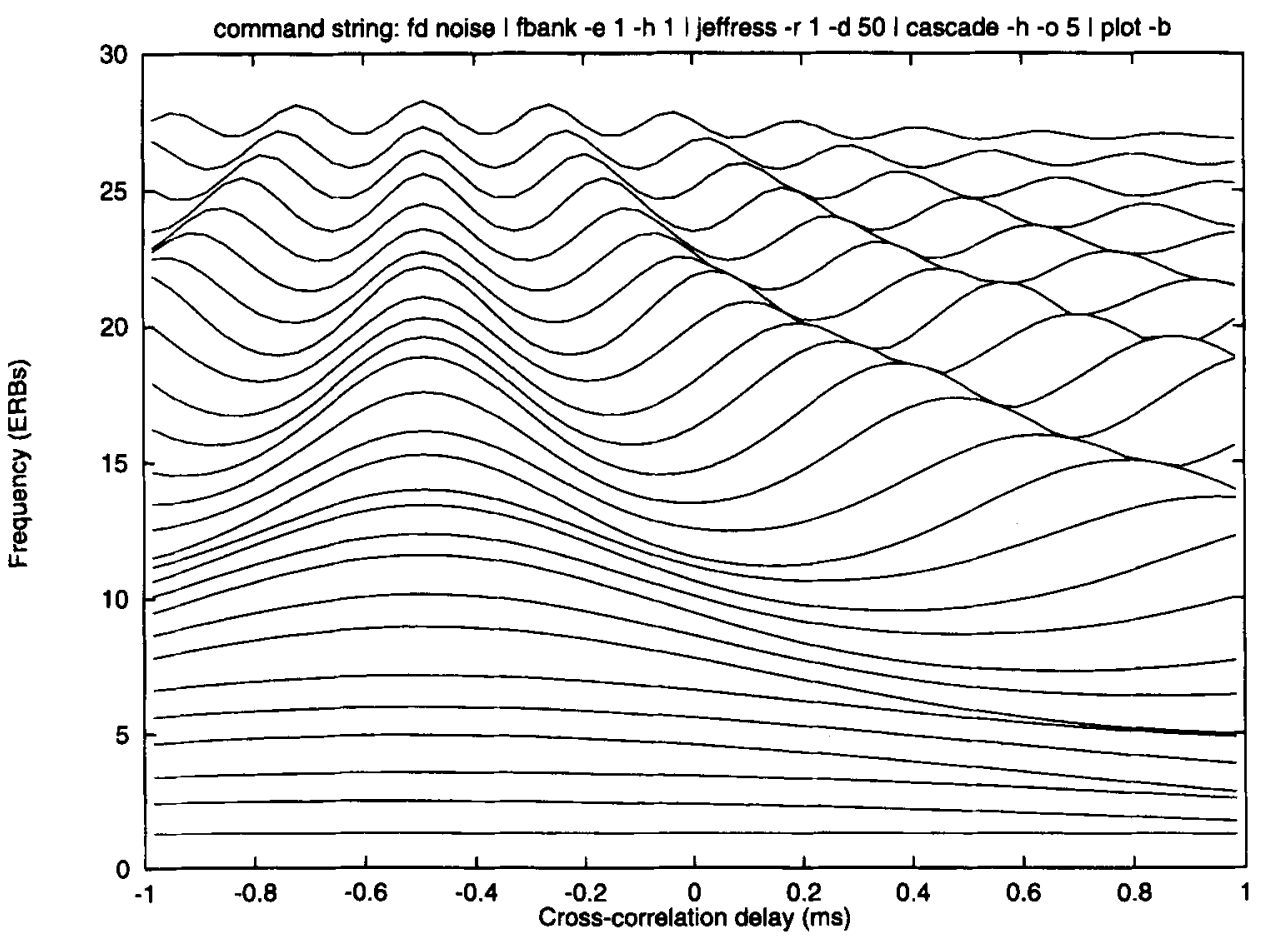

Figure 4. A 30-kHz stereo waveform file is processed, as in Figure 3, by a gamma-tone filterbank and haircell model. Frequency channels of equal center frequency but from different stereo channels are then cross-correlated (running cross-correlation with 50-msec time constant) to simulate binaural processing (Jeffress, 1972). As in Figure 3, the resulting waveforms are plotted in a cascade. The ridge of corresponding peaks across the different functions reveals that the left-hand input channel was delayed with respect to the right-hand channel by $500 \mu \mathrm{sec}$. Execution time, $121 \mathrm{sec}$. 


\section{Availability and Documentation}

All of the software described is available without charge to academic users to use and modify, but not to redistribute. The source, manual pages, and so on, can be accessed via anonymous ftp from ftp.ihr.mrc.ac.uk in $\sim \mathrm{ftp} / \mathrm{pub} / \mathrm{johncu} /$ wave.tar.Z. This 2.5 -MB compressed archive is over $5 \mathrm{MB}$ when uncompressed. The author may be contacted by e-mail (jfc@physiol.ox.ac.uk) regarding difficulties in transfer, compilation, or use. Although/WAVE is free, some of the software was written by other authors and has been included in order to provide as wide a range of facilities as possible and also to ensure that everything necessary to the user is provided in one package. These pieces of software are mostly located in their own directories and carry their own copyright declarations. In a few cases, such software has been included in the main source directory and carries copyright declarations in the source code. The software is all written in C. Comprehensive UNIX manual pages and some tutorial documents are included.

\section{REFERENCES}

ABED, A. H. M., \& CAIN, G. D. (1978). Low-pass digital filtering with the host windowing design technique. Radio \& Electronic Engineer, 48, 293-300.

Abed, A. H. M., \& CaIN, G. D. (1984). Host windowing technique for FIR digital filter design. IEEE Transactions on Acoustics, Speech \& Signal Processing, 32, 683-684.

Bechtel, W., \& Abrahamson, A. (1991). Connectionism and the mind: An introduction to parallel processing in networks. Oxford: Blackwell.

ColbuRN, H. S. (1973). Theory of binaural interaction based on auditory-nerve data. I. General strategy and preliminary results on interaural discrimination. Journal of the Acoustical Society of America, 54, 1458-1470.

ColburN, H. S. (1977). Theory of binaural interaction based on auditory-nerve data. II. Detection of tones in noise. Journal of the Acoustical Society of America, 61, 525-533.

Culling, J. F., \& Darwin, C. J. (1994). Perceptual and computational separation of simultaneous vowels: Cues arising from lowfrequency beating. Journal of the Acoustical Society of America, 95, 1559-1569.

Culling, J. F., \& Summerfield, Q. (1995). Perceptual separation of concurrent speech sounds: Absence of across-frequency grouping by common interaural delay. Journal of the Acoustical Society of America, 98, 785-797.

Culling, J. F., Summerfield, Q., \& Marshall, D. H. (1994). Effects of simulated reverberation on the use of binaural cues and fundamental frequency differences for separating concurrent vowels. Speech Communication, 14, 71-95.

DuRLaCh, N. I. (1960). Note on the equalization-cancellation theory of binaural masking level differences. Journal of the Acoustical Society of America, 32, 1075-1076.

DuRLACH, N. I. (1972). Binaural signal detection: Equalization and cancellation theory. In J. V. Tobias (Ed.), Foundations of modern auditory theory (Vol. 2, pp. 371-462). New York: Academic Press.

JEFFRESS, L. A. (1972). Binaural signal detection: Vector theory. In
J. V. Tobias (Ed.), Foundations of modern auditory theory (Vol. 2, pp. 351-367). New York: Academic Press.

Kernighan, B. W., \& Ritchie, D. M. (1978). The C programming language. Englewood Cliffs, NJ: Prentice-Hall.

KiATt, D. H. (1980). Software for a parallel/cascade formant synthesizer. Journal of the Acoustical Society of America, 67, 838-844.

LickLIDER, J. C. R. (1951). A duplex theory of pitch perception. Experientia, 7, 128-133.

MEDDIS, R. (1986). Simulation of mechanical to neural transduction in the auditory receptor. Journal of the Acoustical Society of America, 79, 702-711.

MEDDIS, R. (1988). Simulation of auditory transduction; Further studies. Journal of the Acoustical Society of America, 83, 1056-1063.

MEDDIS, R., \& HewITr, M. (1991a). Virtual pitch and phase sensitivity of a computer model of the auditory periphery. I: Pitch identification. Journal of the Acoustical Society of America, 89, 2866-2882.

MEDDIs, R., \& HEwITT, M. (1991b). Virtual pitch and phase sensitivity of a computer model of the auditory periphery. II: Phase sensitivity. Journal of the Acoustical Society of America, 89, 2883-2894.

MoORE, B. C. J., \& GlasberG, B. R. (1983). Suggested formulae for calculating auditory-filter bandwidths and excitation patterns. Journal of the Acoustical Society of America, 74, 750-753.

Moore, B. C. J., \& GlasBerG, B. R. (1987). Formulae describing frequency selectivity as a function of frequency and level, and their use in excitation patterns. Hearing Research, 28, 209-225.

Patterson, R. D., \& Moore, B. C. J. (1986). Auditory filters and excitation patterns as representations of frequency resolution. In B. C. J. Moore (Ed.), Frequency selectivity in hearing (pp. 123177). London: Academic Press.

Patterson, R. D., Nimmo-Smith, I., Holdsworth, J., \& Rice, P. (1987, December). An efficient auditory filterbank based on the gamma-tone function. Paper presented to the Institute of Acoustics speech group on auditory modelling at the Royal Signals Research Establishment.

Patterson, R. D., Nimmo-Smith, I., Holdsworth, J., \& Rice, P. (1988). Spiral VOS final report, Part A: The auditory filterbank (Cambridge Electronic Design Contract Rep. APU 2341). Unpublished document.

Perlman, G. (1980). Data analysis programs for the UNIX operating system. Behavior Research Methods \& Instrumentation, 12, 554-558.

Peterson, P. M. (1986). Simulating the response of multiple microphones to a single acoustic source in a reverberant room. Journal of the Acoustical Society of America, 80, 1527-1529.

Plack, C. J., \& Moore, B. C. J. (1990). Temporal window shape as a function of frequency and level. Journal of the Acoustical Society of America, 88, 2178-2187.

RABINER, L. R., \& GoLD, B. (1975). Theory and application of digital signal processing. Englewood Cliffs, NJ: Prentice-Hall.

Shackleton, T. M., Meddis, R., \& Hewitt, M. J. (1992). Acrossfrequency integration in a model of lateralization. Journal of the Acoustical Society of America, 91, 2276-2279.

TRINDER, J. R. (1982). Hardware-software configuration for high performance digital filtering in real time. Proceedings of the International Conference on Acoustics, Speech \& Signal Processing, 2, 687-690.

\section{NOTE}

1. $w d m$ is essentially identical to $d m$, described by Perlman (1980), except that it reads and writes header information before the data. Perlman's code is included during compilation.

(Manuscript received January 3, 1995; revision accepted for publication May 26, 1995.) 